\title{
THE MIXED HODGE STRUCTURE ON THE FUNDAMENTAL GROUP OF THE FIBER TYPE 2-ARRANGEMENT
}

\author{
YUKIHITO KAWAHARA
}

\begin{abstract}
The complement of an arrangement of hyperplanes is a good example of the mixed Hodge structure on the fundamental group of an algebraic variety. We compute its isomorphic class using iterated integrals in the fiber type case and then get the combinatorial and projective invariant.
\end{abstract}

\section{Introduction}

The mixed Hodge structure on the homotopy group of the algebraic variety was constructed in two different ways. One way is Morgan's construction based on Sullivan's theory of minimal models [M]. The other way is Hain's method based on the bar construction [H5]. We shall deal with Hain's method as this approach is very natural from the topological viewpoint and it directly gives precise results on the fundamental group.

Due to Hain [H1], we can construct a mixed Hodge structure on the fundamental group of an algebraic variety using iterated integrals defined by $\mathrm{K}-\mathrm{T}$. Chen as follows. Let $V$ be an algebraic variety over $\mathbb{C}$. We fix a point $x$ of $V$ and consider the truncation

$$
\mathbb{Z} \pi_{1}(V, x) / J^{s+1}
$$

of the group algebra of the fundamental group $\pi_{1}(V, x)$ over $\mathbb{Z}$ by some power of its augmentation ideal $J$. An iterated integral is a function on the space of paths in $V$. Let us denote the space of iterated integrals with length $\leqq s$ that are homotopy functionals on the space of loops based at $x$ (i.e. its value depends only on the homotopy class of the loop.), by

$$
H^{0}\left(B_{s}(V), x\right) \text {. }
$$

Then the integral map

$$
H^{0}\left(B_{s}(V), x\right) \rightarrow \operatorname{Hom}_{\mathbb{Z}}\left(\mathbb{Z} \pi_{1}(V, x) / J^{s+1}, \mathbb{C}\right)
$$

Received May 25, 1995. 
is an isomorphism (K-T. Chen). As an iterated integral $\int \omega_{1} \cdots \omega_{r}$ is in $F^{p}$ if the total number of $d$ 's in $\omega_{j}$ 's is $\geqq p$, a Hodge filtration on $H^{0}\left(B_{s}(V), x\right)$ can be defined. The weight filtration is given by the length filtration when $V$ is smooth and projective:

$$
W_{l}\left(\mathbb{Z} \pi_{1}(V, x) / J^{s+1}\right)^{*}=\left(\mathbb{Z} \pi_{1}(V, x) / J^{l+1}\right)^{*} \cong H^{0}\left(B_{l}(V), x\right)
$$

If $H^{1}(V)$ is a pure Hodge structure of weight 2, then the weight filtration on $\mathbb{Z} \pi_{1}(V, x) / J^{s+1}$ is defined by

$$
W_{2 l+1}=W_{2 l}=\left(\mathbb{Z} \pi_{1}(V, x) / J^{l+1}\right)^{*} \cong H^{0}\left(B_{l}(V), x\right) .
$$

Thus the Hodge and weight filtration induced by those define a mixed Hodge structure on $\mathbb{Z} \pi_{1}(V, x) / J^{s+1}$.

In particular, when $s=1$, there is an isomorphism

$$
\mathbb{C} \pi_{1}(V, x) / J^{2} \cong \mathbb{C} \oplus H_{1}(V, \mathbb{C})
$$

of mixed Hodge structures. And, since the mixed Hodge structure on $H_{1}(V)$ is independent of the base point, so is the same on $\mathbb{C} \pi_{1}(V, x) / J^{2}$. Thus, the interesting case is when $s=2$; the mixed Hodge structure on $\mathbb{C} \pi_{1}(V, x) / J^{3}$ will vary with the base point. In fact,

THEOREM. If $V=\mathbb{P}^{1}-\left\{a_{1}, \cdots, a_{n}\right\}$, then the polarized mixed Hodge structure on $J(V, t) / J^{3}$ determines $(V, t)$ up to biholomorphism.

And if $V$ is a smooth projective algebraic curve, the similar theorem is obtained by Hain and Pulte $([\mathrm{H} 1])$.

On the other hand $\mathbb{P}^{1}-\left\{a_{1}, \cdots, a_{n}\right\}$ can be seen as the complement of an arrangement of hyperplanes in $\mathbb{C}$. We consider the same arguments in the case of the complement of some 2-arrangement. An arrangement $\mathcal{A}$ of hyperplanes in $\mathbb{C}^{2}$ is called fiber type if

$$
\mathcal{A}=\left\{H_{1}, \cdots, H_{n}, G_{1}, \cdots, G_{m}\right\}
$$

satisfies the following conditions.

(1) Each $G_{j}$ is parallel to $G_{1}$, and each $H_{i}$ is not parallel to $G_{1}$. (i.e. For each $i \neq j, G_{i} \cap G_{j}=\emptyset$ and for each $\left.k, l, H_{k} \cap G_{l} \neq \emptyset.\right)$

(2) If $H_{i} \cap H_{j} \neq \emptyset$, then there exists unique $G_{k}$ such that

$$
H_{\imath} \cap H_{\jmath} \subset G_{k}
$$


In general, if $H_{1}(V, \mathbb{Z})$ is torsionfree, there is an exact sequence

$$
0 \longrightarrow H^{1}(V) \longrightarrow \operatorname{Hom}\left(J / J^{3}, \mathbb{Z}\right) \longrightarrow K \longrightarrow 0
$$

where $K$ is the kernel of the cup product $H^{1}(V) \otimes H^{1}(V) \rightarrow H^{2}(V)$. When $H^{1}(V)$ has a pure Hodge structure, the mixed Hodge structure on the dual of $J / J^{3}$ is a separated extension of Hodge structures. The set of suitable classes of extensions of $K$ by $H^{1}$ forms an abelian group $\operatorname{Ext}\left(K, H^{1}\right)$, and there is an abelian group isomorphism (see [Ca])

$$
\psi: \operatorname{Ext}\left(K, H^{1}\right) \rightarrow \frac{\operatorname{Hom}\left(K, H^{1}\right)_{\mathbb{C}}}{F^{0} \operatorname{Hom}\left(K, H^{1}\right)_{\mathbb{C}}+\operatorname{Hom}\left(K, H^{1}\right)_{\mathbb{Z}}} .
$$

For a pointed fiber type 2-arrangement $(\mathcal{A}, b)$, with suitable each basis of $H^{1}$ and $K$, the concrete description of $\psi\left(\left(J\left(M(\mathcal{A}, b) / J^{3}\right)^{*}\right)\right.$ gives that it depends only on cross ratios

$$
\lambda_{i j} \quad(1 \leqq i<j \leqq n) \quad \lambda_{\imath \jmath}^{\prime} \quad(1 \leqq i<j \leqq m)
$$

arising from $(\mathcal{A}, b)$. Two pointed fiber type 2-arrangements are called cross ratio equivalent if these respective cross ratios coincide. Then we obtain the following result.

Main Theorem. Let $(\mathcal{A}, b)$ and $\left(\mathcal{A}^{\prime}, b^{\prime}\right)$ be pointed fiber type 2arrangements. If there is a ring isomorphism

$$
\varphi: \mathbb{Z} \pi_{1}(M(\mathcal{A}), b) / J^{3} \rightarrow \mathbb{Z} \pi_{1}\left(M\left(\mathcal{A}^{\prime}\right), b^{\prime}\right) / J^{3}
$$

that induces an isomorphism of mixed Hodge structures, then $(\mathcal{A}, b)$ and $\left(\mathcal{A}^{\prime}, b^{\prime}\right)$ are cross ratio equivalent.

\section{$\S 1$. The mixed Hodge structure on $\pi_{1}$}

In this section, we review some results on the mixed Hodge structure on $\pi_{1}$ using Hain's method [H1]. 


\section{The mixed Hodge structure on $\pi_{1}$}

Let $k=\mathbb{R}$ or $\mathbb{C}$, and $M$ a smooth manifold. Denote the set of piecewise smooth path $\gamma:[0,1] \rightarrow M$ by $P M$ and the subset of loops based at $x$ by $P_{x} M . E_{k}(M)$ denotes the de Rham complex of $C^{\infty} k$-valued forms on $M$. First we denote the iterated integral as follows. For $\omega_{1}, \ldots, \omega_{r} \in E_{k}^{1}(M)$ and $\gamma \in P M$, define

$$
\int_{\gamma} \omega_{1} \cdots \omega_{r}=\int \cdots \int_{0 \leqq t_{1} \leqq \cdots \leqq t_{r} \leqq 1} f_{1}\left(t_{1}\right) \cdots f_{r}\left(t_{r}\right) d t_{1} \cdots d t_{r},
$$

where $f_{j}(t) d t=\gamma^{*} \omega_{j} . \quad \int \omega_{1} \cdots \omega_{r}$ denotes the function $P M \rightarrow k, \gamma \rightarrow$ $\int_{\gamma} \omega_{1} \cdots \omega_{r}$. If $r=0$, it is the constant function. A linear combination of such functions and the constant function is called an iterated path integral, and a linear combination of a constant function and iterated integrals $\int \omega_{1} \cdots \omega_{r}$ with $r \leqq s$ is called an iterated integral of length $\leqq s$. Sometime we shall denote the integration value of an iterated integral $I$ on a path $\gamma$ by $\langle I, \gamma\rangle$.

A function $F$ on $P M$ is homotopy functional, if $F(\gamma)$ depends only on the homotopy class of $\gamma$ relative to its endpoints.

We define subspaces of iterated integrals;

$B_{s}(M)=\{$ iterated integrals on $M$ of length $\leqq s\}$,

$H^{0}\left(B_{s}(M), x\right)=\left\{I \in B_{s}(M) \mid I\right.$ is homotopy functional on $\left.P_{x} M\right\}$,

$\bar{B}_{s}(M)=\left\{I \in B_{s}(M)\right.$ with zero constant term $\}$, $H^{0}\left(\bar{B}_{s}(M), x\right)=H^{0}\left(B_{s}(M), x\right) \cap \bar{B}_{s}(M)$.

Note that

$$
B_{s}(M)=k \oplus \bar{B}_{s}(M) .
$$

Suppose that $A$ is a subdifferential graded algebra of $E_{k}(M)$ such that the inclusion $A^{\cdot} \rightarrow E_{k}(M)$ is a quasi isomorphism. $B_{s}\left(A^{\cdot}\right)$ is a set of iterated integrals on $M$ spanned by $\int \omega_{1} \cdots \omega_{r}$ where each $\omega_{j} \in A^{1}$ and $0 \leqq r \leqq s$. We define subspaces $H^{0}\left(B_{s}\left(A^{\cdot}\right), x\right), \bar{B}_{s}\left(A^{\cdot}\right)$, and $H^{0}\left(\bar{B}_{s}\left(A^{*}\right), x\right)$ in the same way.

Let $G$ be a group and $R$ a commutative ring. We denote by $R G$ the group algebra of $G$ over $R$ and by $J$ its augmentation ideal.

Theorem 1.1. (Chen) For each $x \in M$ and $s>0$, the integration map

$$
H^{0}\left(B_{s}\left(A^{\cdot}\right), x\right) \rightarrow \operatorname{Hom}_{\mathbb{Z}}\left(\mathbb{Z} \pi_{1}(M, x) / J^{s+1}, k\right)
$$

is an isomorphism. 
Proof. The proof is given in $[\mathrm{H} 1,2,3]$. cf. $\left.{ }_{\perp} \mathrm{C} 1,2,3\right]$.

Corollary 1.2 .

$$
H^{0}\left(\bar{B}_{s}\left(A^{\cdot}\right), x\right) \rightarrow \operatorname{Hom}_{\mathbb{Z}}\left(J(M, x) / J^{s+1}, k\right)
$$

is an isomorphism.

Using this, we can give the mixed Hodge structure on $\mathbb{Z} \pi_{1}(M, x) / J^{s+1}$.

TheOREM 1.3. (Morgan, Hain) If $M$ is an algebraic variety over $\mathbb{C}$ and $x \in V$, then there is a mixed Hodge structure on

$$
\mathbb{Z} \pi_{1}(M, x) / J^{s+1}
$$

that is natural with respect to morphism of pointed varieties. Moreover, if $s \geqq t$, then the quotient map

$$
\mathbb{Z} \pi_{1}(M, x) / J^{s+1} \rightarrow \mathbb{Z} \pi_{1}(M, x) / J^{t+1}
$$

induces a morphism of mixed Hodge structures.

Proof. The theorem is proved by induction $s$, using the following proposition. For detail, see [H1].

Proposition 1.4. (Hain) There is a natural isomorphism

$$
\begin{aligned}
\mathbb{C} \oplus H^{1}(M, \mathbb{C}) & \rightarrow H^{0}\left(B_{1}(M), x\right) \\
\lambda \oplus \omega & \rightarrow \lambda+\int \omega
\end{aligned}
$$

and for all $s$, the sequence

$$
0 \longrightarrow H^{0}\left(B_{s-1}(M), x\right) \longrightarrow H^{0}\left(B_{s}(M), x\right) \stackrel{p}{\longrightarrow} \otimes^{s} H^{1}(M, \mathbb{C})
$$

is exact, where $p$ takes the iterated integral I to the function

$$
\begin{aligned}
& p(I): \otimes^{s} H_{1}(M, \mathbb{C}) \longrightarrow \mathbb{C} \\
& {\left[\alpha_{1}\right] \otimes \cdots \otimes\left[\alpha_{s}\right] \longrightarrow<I, \prod_{j=1}^{s}\left(\alpha_{\jmath}-1\right)>}
\end{aligned}
$$

for each loop $\alpha_{j}$ based at $x$.

Proof. The proof is given in [H1]. 


\section{Extensions of mixed Hodge structures}

We also review the extension of mixed Hodge structures, (cf. [Ca] and $[\mathrm{H} 1])$.

A separated extension of Hodge structures is an exact sequence

$$
0 \rightarrow A \rightarrow E \rightarrow B \rightarrow 0
$$

of mixed Hodge structures, where $A$ is a pure Hodge structure of weight $m$, $B$ is a pure Hodge structure of weight $n$, and $n>m$. Two extensions

$$
0 \rightarrow A \rightarrow E_{j} \rightarrow B \rightarrow 0, \quad j=1,2
$$

are congruent if there is an isomorphism of mixed Hodge structures $\Phi$ : $E_{1} \rightarrow E_{2}$ such that

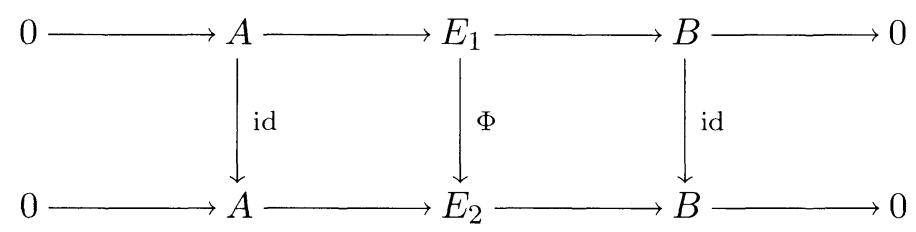

commutes. The set of congruence classes of extensions of $B$ by $A$ forms an abelian group that we shall denote by $\operatorname{Ext}(B, A)$. There is an abelian group isomorphism

$$
\psi: \operatorname{Ext}(B, A) \rightarrow \frac{\operatorname{Hom}(B, A)_{\mathbb{C}}}{F^{0} \operatorname{Hom}(B, A)_{\mathbb{C}}+\operatorname{Hom}(B, A)_{\mathbb{Z}}}
$$

that is given as follows. If

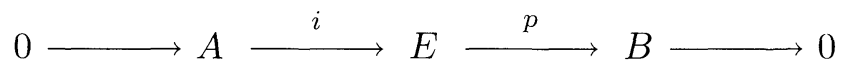

is an extension, choose a Hodge filtration preserving section $s_{F}: B \rightarrow E$ of $p$ and a retraction $r_{\mathbb{Z}}: E \rightarrow A$ of $i$ that is defined over $\mathbb{Z}$. Composing these gives an element $\psi(E)=r_{\mathbb{Z}} \circ s_{F}$ of $\operatorname{Hom}(B, A)_{\mathbb{C}}$. It can be checked that $\psi(E)=r_{\mathbb{Z}} \circ s_{F}$ is well-defined modulo $F^{0} \operatorname{Hom}(B, A)_{\mathbb{C}}+\operatorname{Hom}(B, A)_{\mathbb{Z}}$. It is easy to construct an inverse of $\xi$. For detail see [Ca].

When $B$ is of weight $2 p$, then $r_{\mathbb{Z}} \circ s_{F}$ also induces a homomorphism 


$$
\mu: B_{\mathbb{Z}}^{p, p} \rightarrow A_{\mathbb{C}} / F^{p} A+A_{\mathbb{Z}}
$$

called the motif of the extension (cf. [Ca]) where $B_{\mathbb{Z}}^{p, p}=B_{\mathbb{Z}} \cap B^{p, p}$.

We can express the mixed Hodge structure on $\left(J / J^{3}\right)^{*}$ as an extension.

Lemma 1.5. Suppose that $(X, x)$ is a path connected, pointed topological space. If $H_{1}(X, \mathbb{Z})$ is torsion-free, then there is an exact sequence

$$
0 \longrightarrow H_{\mathbb{Z}}^{1}(X) \stackrel{i}{\longrightarrow} \operatorname{Hom}_{\mathbb{Z}}\left(J(X, x) / J^{3}, \mathbb{Z}\right) \stackrel{p}{\longrightarrow} H_{\mathbb{Z}}^{1}(X) \otimes H_{\mathbb{Z}}^{1}(X) \longrightarrow H_{\mathbb{Z}}^{2}(X) .
$$

Here $i(z)(g-1)=\langle z, g\rangle$, where $g \in \pi_{1}(X, x)$ and $z \in H^{1}(X)$. If $\phi \in$ $\left(J / J^{3}\right)^{*}$ and $\alpha, \beta$ are loops based at $x$, then

$$
p(\phi)([\alpha] \otimes[\beta])=<\phi,(\{\alpha\}-1)(\{\beta\}-1)>.
$$

Proof. See [H1].

\section{$\S 2$. Fiber type arrangements}

Definition 2.1. Let $\mathcal{A}$ be 2-arrangement i.e. a finite set of hyperplanes in $\mathbb{C}^{2}$ and $M(\mathcal{A})$ a complement of $\mathcal{A}$ in $\mathbb{C}^{2}$. We call $\mathcal{A}$ affine fiber type if $\mathcal{A}$ is a set $\left\{H_{1}, \cdots, H_{n}, G_{1}, \cdots, G_{m}\right\}$ of hyperplanes in $\mathbb{C}^{2}$ satisfying the following conditions

(1) Each $G_{j}$ is parallel to $G_{1}$ and each $H_{i}$ is not parallel to $G_{1}$. (i.e. For each $i \neq j, G_{i} \cap G_{\jmath}=\emptyset$ and for each $k, l, H_{k} \cap G_{l} \neq \emptyset$.)

(2) If $H_{\imath} \cap H_{j} \neq \emptyset$, then there exists unique $G_{k}$ such that

$$
H_{i} \cap H_{j} \subset G_{k}
$$

We assume that all $H_{i}$ 's are not parallel each other. (In this paper arrangements mean affine arrangements.), cf. see $[\mathrm{FR}]$ and $[\mathrm{J}]$.

Now we reconsider the extension of $\left(J / J^{3}\right)^{*}$. For a fiber type 2-arrangement $\mathcal{A}$ and a base point $b$ of the complement $M=M(\mathcal{A})$ of $\mathcal{A}$, there is the extension

$$
0 \longrightarrow H^{1}(M) \longrightarrow\left(J / J^{3}\right)^{*} \longrightarrow 0
$$


of $\left(J / J^{3}\right)^{*}$ where $J$ is the augmentation ideal of the group algebra of $\pi_{1}(M(\mathcal{A}), b)$ over $\mathbb{Z}$ and $K$ is the kernel of the cup-product $H^{1}(M) \otimes$ $H^{1}(M) \rightarrow H^{2}(M)$. Since the first cohomology is pure of weight 2 and the kernel $K$ of its cup product is pure of weight 4 , there is the extension isomorphism

$$
\psi: \operatorname{Ext}\left(K, H^{1}\right) \rightarrow \operatorname{Hom}\left(K, H^{1}\right)_{\mathbb{C}} / \operatorname{Hom}\left(K, H^{1}\right)_{\mathbb{Z}}
$$

We shall give the description of $\psi\left(\left(J / J^{3}\right)^{*}\right)$ for $(\mathcal{A}, b)$. First each basis of $H^{1}(M), H_{1}(M), K$ and $K^{*}$ can be given as follows.

As we take a coordinate $(x, y)$ in $\mathbb{C}^{2}$, we can assume that

$$
\mathcal{A}=\left\{H_{1}, \cdots, H_{n}, G_{1}, \cdots, G_{m}\right\}
$$

where each $H_{i}$ is defined by the equation $y=h_{i}(x)$ and each $G_{j}$ is defined by the equation $g_{j}(x)=0$ where $h_{i}, g_{j}$ are linear polynomials in $x$. Set

$$
\omega_{i}=\frac{1}{2 \pi \sqrt{-1}} d \log \left(y-h_{\imath}(x)\right) \quad 1 \leqq i \leqq n
$$

and

$$
\eta_{j}=\frac{1}{2 \pi \sqrt{-1}} d \log \left(g_{\jmath}(x)\right) \quad 1 \leqq j \leqq m .
$$

Brieskorn showed that the cohomology of the complement of hyperplanes $H_{k}=\left\{l_{k}=0\right\}$ is generated by forms

$$
w_{k}=\frac{1}{2 \pi \sqrt{-1}} d \log \left(l_{k}\right)
$$

in general $[\mathrm{B}]($ for detail, see $[\mathrm{OT}: 5.4])$. Then $\omega_{i}$ 's and $\eta_{j}$ 's generate the cohomology $H^{*}(M(\mathcal{A}), \mathbb{Z})$. Set

$$
B H^{1}=\left\{\omega_{1}, \cdots, \omega_{n}, \eta_{1}, \cdots, \eta_{m}\right\} .
$$

Let $\mathcal{B}$ be the subarrangement $\left\{G_{1} \cdots, G_{m}\right\}$ of $\mathcal{A}$. Fix a base point $b=\left(x_{0}, y_{0}\right)$ of $M(\mathcal{A})$. We put

$$
F=\left\{\left(x_{0}, y\right) \in M(\mathcal{A})\right\}=\mathbb{C}-\left\{y_{1}, \cdots, y_{n}\right\}
$$

and

$$
B=\left\{\left(x, y_{0}\right) \in M(\mathcal{B})\right\}=\mathbb{C}-\left\{x_{1}, \cdots, x_{m}\right\}
$$


where

$$
y_{\imath}=h_{i}\left(x_{0}\right) \quad 1 \leqq i \leqq n
$$

and

$$
x_{j}=\operatorname{Ker} g_{\jmath} \quad 1 \leqq j \leqq m .
$$

Choose loops $\alpha_{i}, 1 \leqq i \leqq n$, based at $y_{0}$ in $F$ such that, for each $i, \alpha_{i}$ is anti-clockwise around $y_{i}$ and nullhomotopic in $\mathbb{C}-\left\{y_{1}, \cdots, \check{y}_{i}, \cdots, y_{n}\right\}$. In the same way we choose loops $\beta_{j}, 1 \leqq j \leqq m$ based at $x_{0}$ in $B$. It is clear that

$$
\int_{\alpha_{\imath}} \omega_{j}=\delta_{i j} \quad \int_{\beta_{\imath}} \eta_{j}=\delta_{i j} \quad \int_{\alpha_{\imath}} \eta_{j}=\int_{\beta_{i}} \omega_{j}=0
$$

where $\delta_{i j}$ is Kronecker's delta. Consequently, $\left[\alpha_{1}\right], \cdots,\left[\alpha_{n}\right],\left[\beta_{1}\right], \cdots,\left[\beta_{m}\right]$ is the dual basis of $H_{1}(M(\mathcal{A}), \mathbb{Z})$. Set

$$
B H_{1}=\left\{\alpha_{1}, \cdots, \alpha_{n}, \beta_{1}, \cdots, \beta_{m}\right\}
$$

Remark 2.2. In general, let $\mathcal{A}$ be an arrangement of hyperplanes in $\mathbb{C}^{N}$ and $M=M(\mathcal{A})$ the complement of $\mathcal{A}$. The mixed Hodge structure on the cohomology $H^{\imath}(M)$ is pure. Moreover any element of $H^{i}(M)$ has the Hodge type $(i, i)$ (see $[\mathrm{Sh}])$.

We hope to find the basis of $K$. In general, if a vector space $H$ of dimension $n$ has a basis $\tau_{1}, \cdots, \tau_{n}$, then we can choose the basis of $H \otimes H$ as

$$
\begin{aligned}
\frac{1}{2}\left[\tau_{i}, \tau_{j}\right]=\frac{1}{2}\left(\tau_{i} \tau_{j}-\tau_{j} \tau_{i}\right) & 1 \leqq i<j \leqq n \\
\frac{1}{2}\left\{\tau_{i}, \tau_{j}\right\}=\frac{1}{2}\left(\tau_{i} \tau_{j}+\tau_{j} \tau_{i}\right) & 1 \leqq i \leqq j \leqq n .
\end{aligned}
$$

We put

$$
\begin{aligned}
& {[\omega]_{i j}= \begin{cases}{\left[\omega_{i}, \omega_{j}\right]+\left[\omega_{j}, \eta_{k}\right]+\left[\eta_{k}, \omega_{i}\right]} & \text { if } \emptyset \neq H_{i} \cap H_{j} \subset G_{k} \\
{\left[\omega_{i}, \omega_{j}\right]} & \text { if } H_{\imath} \cap H_{j}=\emptyset\end{cases} } \\
& {[\eta]_{i j}=\left[\eta_{i}, \eta_{j}\right]} \\
& \{\omega\}_{i j}=\left\{\omega_{i}, \omega_{j}\right\} \\
& \{\eta\}_{i j}=\left\{\eta_{i}, \eta_{j}\right\} .
\end{aligned}
$$

We can obtain the following proposition. 
Proposition 2.3.

$$
B K_{\mathbb{Q}}=\left\{\begin{array}{ll}
\frac{1}{2}[\omega]_{i j} & 1 \leqq i<j \leqq n \\
\frac{1}{2}[\eta]_{i j} & 1 \leqq i<j \leqq m \\
\frac{1}{2}\{\omega\}_{i j} & 1 \leqq i \leqq j \leqq n \\
\frac{1}{2}\{\eta\}_{i j} & 1 \leqq i \leqq j \leqq m \\
\frac{1}{2}\left\{\omega_{i}, \eta_{\jmath}\right\} & 1 \leqq i \leqq n, 1 \leqq j \leqq m
\end{array}\right\}
$$

is a basis of $K_{\mathbb{Q}}$.

Let $\mathcal{H}=\left\{F_{1}, \cdots, F_{l}\right\}$ be an arrangement with $F_{i}=k e r \varphi_{i}$. We define a basis $\tau_{1}, \cdots, \tau_{l}$ of $H^{1}(M(\mathcal{H}))$ by $\tau_{i}=\frac{1}{2 \pi \sqrt{-1}} d \log \varphi_{i}$. Let $Z_{1}, \cdots, Z_{l}$ be the dual basis of $H_{1}(M(\mathcal{H}))$. Denote the free associative algebra they generate by $\mathbb{C}<Z_{1}, \cdots, Z_{l}>$. We shall denote its augmentation ideal by $I$. The geometric lattice $L(\mathcal{H})$ consists of the subspaces of the form

$$
F_{i_{1}} \cap \cdots \cap F_{i_{p}} \quad \text { where }\left\{i_{1}, \cdots, i_{p}\right\} \subset\{1, \cdots, l\} .
$$

Let $L^{2}(\mathcal{H})$ be a set of codimension-two elements of $L(\mathcal{H})$. For $K \in L^{2}(\mathcal{H})$, we set $\mathcal{H}_{K}=\left\{F_{\imath} \in \mathcal{H} \mid K \subset F_{i}\right\}$ and define the relation ideal $R_{K}$ of $\mathbb{C}<$ $Z_{1}, \cdots, Z_{l}>$ by

$$
\left[Z_{i_{\nu}}, Z_{i_{1}}+\cdots+Z_{i_{p}}\right]=0 \quad 1 \leqq \nu \leqq p
$$

where $\mathcal{H}_{K}=\left\{F_{i_{1}}, \cdots, F_{i_{p}}\right\}$. Let $R=\left(R_{K}\right)_{K \in L^{2}(\mathcal{H})}$ and define

$$
A_{s}=\mathbb{C}<Z_{1}, \cdots, Z_{l}>/ R+I^{s+1} .
$$

Set

$$
\omega=\omega_{1} Z_{1}+\cdots+\omega_{l} Z_{l} \quad \in H^{1}(M(\mathcal{H})) \otimes A_{s}
$$

The relations guarantee that $\omega \wedge \omega=0$. For detail, see $[K 1,2,3]$.

Proof of Proposition 2.3. Let $\mathcal{A}$ be the fiber type 2-arrangement and $M=M(\mathcal{A})$ its complement. We take the basis $\omega_{1}, \cdots, \omega_{n}, \eta_{1}, \cdots, \eta_{m}$ of $H^{1}(M)$ and let $X_{1}, \cdots, X_{n}, Y_{1}, \cdots, Y_{m}$ be a dual basis of $H_{1}(M)$. Set

$$
A_{s}=\mathbb{C}<X_{i}, Y_{j}>/ R+I^{s+1}
$$

and

$$
\tau=\sum \omega_{i} X_{i}+\sum \eta_{\jmath} Y_{j}
$$


where $R$ is the relation ideal for $\mathcal{A}$. Since $\mathcal{A}$ is fiber type, for $K \in L^{2}(\mathcal{A})$, we can write

$$
\mathcal{A}_{K}=\left\{H_{i_{1}}, \cdots, H_{i_{p}}, G_{j_{K}}\right\}
$$

and its relation is

$$
\left[X_{i_{\nu}}, X_{i_{1}}+\cdots+X_{i_{p}}+Y_{j_{K}}\right]=0 \quad 1 \leqq \nu \leqq p
$$

$\Longleftrightarrow$

$$
\left[X_{i_{\nu}}, Y_{j_{K}}\right]=\sum_{\mu=1}^{p}\left[X_{i_{\mu}}, X_{\imath_{\nu}}\right] \quad 1 \leqq \nu \leqq p .
$$

Hence, any $\left[X_{i}, Y_{j}\right]$ is generated by $\left[X_{i}, X_{j}\right]$ 's. For $\emptyset \neq H_{i} \cap H_{\jmath} \subset G_{k}$, there exists uniquely $K_{i j} \in L^{2}(\mathcal{A})$ such that $H_{i}, H_{\jmath}, G_{k} \in \mathcal{A}_{K_{\imath \jmath}}$, and we set

$$
\mathcal{A}_{K_{2 j}}=\left\{H_{i}, H_{j}, G_{k}, H_{i_{1}}, \cdots, H_{i_{p}}\right\} \text {, }
$$

where $i, j, i_{1}, \cdots, i_{p}$ are distinct each other. We can find the relations including terms $\left[X_{i}, X_{j}\right]$;

$$
\begin{aligned}
{\left[X_{i}, Y_{k}\right] } & =\left[X_{j}, X_{i}\right]+\sum_{\mu=1}^{p}\left[X_{i_{\mu}}, X_{i}\right] \\
& =-\left[X_{i}, X_{j}\right]+\sum_{\mu=1}^{p}\left[X_{\imath_{\mu}}, X_{i}\right]
\end{aligned}
$$

and

$$
\left[X_{j}, Y_{k}\right]=\left[X_{i}, X_{j}\right]+\sum_{\mu=1}^{p}\left[X_{\iota_{\mu}}, X_{j}\right] .
$$

Any other relation has no terms of $\left[X_{i}, X_{j}\right]$. Therefore, the coefficient of $\left[X_{i}, X_{j}\right]$ in $\tau \cdot \tau$ is

$$
\frac{1}{2}\left(\left[\omega_{i}, \omega_{j}\right]-\left[\eta_{k}, \omega_{j}\right]+\left[\eta_{k}, \omega_{i}\right]\right)=\frac{1}{2}[\omega]_{i j} .
$$

If $H_{i} \cap H_{j}=\emptyset$, then there is no such relation. The coefficient of $\left[X_{i}, X_{j}\right]$ is only $\frac{1}{2}\left[X_{i}, X_{j}\right]$. Consequently we can write

$$
\begin{aligned}
\tau \cdot \tau= & \sum_{i<j} \frac{1}{2}[\omega]_{i j}\left[X_{i}, X_{j}\right]+\sum_{i<j} \frac{1}{2}[\eta]_{i j}\left[Y_{i}, Y_{\jmath}\right] \\
& +\sum_{i \leqq j} \frac{1}{2}\{\omega\}_{i j}\left\{X_{i}, X_{j}\right\}+\sum_{i \leqq j} \frac{1}{2}\{\eta\}_{i j}\left\{Y_{i}, Y_{j}\right\}+\sum_{i, k} \frac{1}{2}\left\{\omega_{i}, \eta_{k}\right\}\left\{X_{i}, Y_{k}\right\} .
\end{aligned}
$$


Thus $\frac{1}{2}[\omega]_{i j}, \frac{1}{2}[\eta]_{i j}, \frac{1}{2}\{, \quad\}$ is independent in $H^{1} \otimes H^{1}$. Since $\operatorname{dim}\left(H^{2}(M)\right)=$ $m n$, it is a basis of $K$ whose dimension is $(m+n)^{2}-m n$.

Then $\left\{(\gamma-1) \mid \gamma \in B H_{1}\right\}$ is a basis of $J / J^{2} \cong H_{1}$ and $B H^{1}$ is its dual basis of $\left(J / J^{2}\right)^{*} \cong H^{1}$. Moreover $B K_{\mathbb{Q}}$ is a basis of $K \cong\left(J^{2} / J^{3}\right)^{*}$. Set

$$
B K_{\mathbb{Q}}^{*}=\left\{\begin{array}{ll}
{\left[\alpha_{i}-1, \alpha_{j}-1\right]} & 1 \leqq i<j \leqq n \\
{\left[\beta_{i}-1, \beta_{j}-1\right]} & 1 \leqq i<j \leqq m \\
\left\{\alpha_{i}-1, \alpha_{j}-1\right\} & 1 \leqq i \leqq j \leqq n \\
\left\{\alpha_{i}-1, \beta_{j}-1\right\} & 1 \leqq i \leqq n, 1 \leqq j \leqq m
\end{array}\right\}
$$

LEMMA 2.4. BK $K_{\mathbb{Q}}^{*}$ is a dual basis of $J^{2} / J^{3} \cong K_{\mathbb{Q}}^{*}$.

Proof. Since $\alpha_{i}, \beta_{j}$ are dual of $\omega_{i}, \eta_{j}$ respectively, it is enough to prove the following lemma.

LEMmA 2.5. Let $M$ be a smooth manifold, $\tau_{1}, \tau_{2}$ smooth 1-forms on $M$ and $\gamma_{1}, \gamma_{2}$ loops based at $x \in M$. Then

$(1)<\int \tau_{1} \tau_{2},\left(\gamma_{1}-1\right)\left(\gamma_{2}-1\right)>=\int_{\gamma_{1}} \tau_{1} \cdot \int_{\gamma_{2}} \tau_{2}$

$(2)<\int \frac{1}{2}\left[\tau_{1}, \tau_{2}\right],\left[\gamma_{1}-1, \gamma_{2}-1\right]>=\int_{\gamma_{1}} \tau_{1} \cdot \int_{\gamma_{2}} \tau_{2}-\int_{\gamma_{2}} \tau_{1} \cdot \int_{\gamma_{1}} \tau_{2}$.

$(3)<\int \frac{1}{2}\left\{\tau_{1}, \tau_{2}\right\},\left\{\gamma_{1}-1, \gamma_{2}-1\right\}>=\int_{\gamma_{1}} \tau_{1} \cdot \int_{\gamma_{2}} \tau_{2}+\int_{\gamma_{2}} \tau_{1} \cdot \int_{\gamma_{1}} \tau_{2}$.

(4) $<\int \frac{1}{2}\left[\tau_{1}, \tau_{2}\right],\left\{\gamma_{1}-1, \gamma_{2}-1\right\}>=<\int \frac{1}{2}\left\{\tau_{1}, \tau_{2}\right\},\left[\gamma_{1}-1, \gamma_{2}-1\right]>=0$.

Proof. (1) See $[\mathrm{H} 1 ;(2.13)(\mathrm{b})]$.

(2) Using (1), we get

$$
\begin{aligned}
<\int \frac{1}{2}\left[\tau_{1}, \tau_{2}\right],\left[\gamma_{1}-1, \gamma_{2}-1\right]>= & \frac{1}{2}\left\{\int_{\gamma_{1}} \tau_{1} \cdot \int_{\gamma_{2}} \tau_{2}-\int_{\gamma_{1}} \tau_{2} \cdot \int_{\gamma_{2}} \tau_{1}\right\} \\
& -\frac{1}{2}\left\{\int_{\gamma_{2}} \tau_{1} \cdot \int_{\gamma_{1}} \tau_{2}-\int_{\gamma_{2}} \tau_{1} \cdot \int_{\gamma_{1}} \tau_{2}\right\} \\
= & \int_{\gamma_{1}} \tau_{1} \cdot \int_{\gamma_{2}} \tau_{2}-\int_{\gamma_{2}} \tau_{1} \cdot \int_{\gamma_{1}} \tau_{2} .
\end{aligned}
$$

Also we obtain (3) and (4) in the similar way. 
It is note that $B K_{\mathbb{Q}}$ is not a basis of $K_{\mathbb{Z}}$. Though we can choose the following basis of $K_{\mathbb{Z}}$. Set

$$
\begin{gathered}
\omega_{i j}= \begin{cases}\omega_{i} \omega_{j}-\eta_{k} \omega_{j}+\eta_{k} \omega_{i} & \text { if } \emptyset \neq H_{i} \cap H_{j} \subset G_{k} \\
\omega_{\imath} \omega_{j} & \text { if } H_{i} \cap H_{j}=\emptyset\end{cases} \\
\eta_{i j}=\eta_{i} \eta_{j}
\end{gathered}
$$

Corollary 2.6 .

$$
B K_{\mathbb{Z}}=\left\{\begin{array}{ll}
\omega_{i j} & 1 \leqq i<j \leqq n \\
\eta_{i j} & 1 \leqq i<j \leqq m \\
\{\omega\}_{i j} & 1 \leqq i<j \leqq n \\
\{\eta\}_{i j} & 1 \leqq i<j \leqq m \\
\left\{\omega_{i}, \eta_{j}\right\} & 1 \leqq i \leqq n, 1 \leqq j \leqq m \\
\omega_{i i}=\omega_{i} \omega_{i} & 1 \leqq i \leqq n \\
\eta_{i i}=\eta_{i} \eta_{i} & 1 \leqq i \leqq m
\end{array}\right\}
$$

is a basis of $K_{\mathbb{Z}}$. And also

$$
B K_{\mathbb{Z}}^{*}=\left\{\begin{array}{ll}
{\left[\alpha_{i}-1, \alpha_{j}-1\right]} & 1 \leqq i<j \leqq n \\
{\left[\beta_{i}-1, \beta_{\jmath}-1\right]} & 1 \leqq i<j \leqq m \\
\left(\alpha_{j}-1\right)\left(\alpha_{i}-1\right) & 1 \leqq i<j \leqq n \\
\left(\beta_{j}-1\right)\left(\beta_{\imath}-1\right) & 1 \leqq i<j \leqq m \\
\left(\alpha_{i}-1\right)\left(\beta_{j}-1\right) & 1 \leqq i \leqq n, 1 \leqq j \leqq m \\
\left(\alpha_{i}-1\right)\left(\alpha_{i}-1\right) & 1 \leqq i \leqq n \\
\left(\beta_{i}-1\right)\left(\beta_{i}-1\right) & 1 \leqq i \leqq m
\end{array}\right\}
$$

is its dual basis of $K_{\mathbb{Z}}^{*}$.

Proof. From Proposition 2.3 it is clear that $B K_{\mathbb{Z}}$ is a basis of $K_{\mathbb{Z}}$. Using lemma 2.5, we can check its duality. For example,

$$
\begin{gathered}
<\int \omega_{i \jmath},\left[\alpha_{i}-1, \alpha_{j}-1\right]>=\int_{\alpha_{\imath}} \omega_{i} \int_{\alpha_{j}} \omega_{j}-\int_{\alpha_{\jmath}} \omega_{i} \int_{\alpha_{\imath}} \omega_{j}=1 \\
<\int \omega_{i \jmath},\left(\alpha_{\jmath}-1\right)\left(\alpha_{i}-1\right)>=\int_{\alpha_{\jmath}} \omega_{i} \int_{\alpha_{i}} \omega_{j}=0 \\
<\int \omega_{i j},\left(\alpha_{s}-1\right)\left(\beta_{t}-1\right)>=\int_{\alpha_{s}} \omega_{i} \int_{\beta_{t}} \omega_{j}-\int_{\alpha_{s}} \eta_{k} \int_{\beta_{t}} \omega_{j}+\int_{\alpha_{s}} \eta_{k} \int_{\beta_{t}} \omega_{\imath}=0 .
\end{gathered}
$$


Consequently, for $(\mathcal{A}, b)$, we give the description of $\psi\left(\left(J / J^{3}\right)^{*}\right)$ by

$$
\begin{gathered}
\psi\left(\left(J / J^{3}\right)^{*}\right)=\sum_{\chi \in B K_{\mathbb{Z}}^{*}, \gamma \in B H_{1}} I(\chi: \gamma) \chi \otimes(\gamma-1)^{*} \bmod \left(K^{*} \otimes H_{1}^{*}\right)_{\mathbb{Z}} \\
\in\left(K^{*} \otimes H_{1}^{*}\right)_{\mathbb{C}} /\left(K^{*} \otimes H_{1}^{*}\right)_{\mathbb{Z}} \cong \operatorname{Hom}\left(K, H^{1}\right)_{\mathbb{C}} / \operatorname{Hom}\left(K, H^{1}\right)_{\mathbb{Z}}
\end{gathered}
$$

where, since a dual of $\chi$ is $\chi^{*} \in B K_{\mathbb{Z}}$, we define

$$
I(\chi: \gamma)=\int_{\gamma} \chi^{*}
$$

\section{$\S 3$. The cross ratio equivalence}

We compute the description of $\psi\left(\left(J / J^{3}\right)^{*}\right)$ for $(\mathcal{A}, b)$. First we prepare the following lemma.

Lemma 3.1. Set

$$
\tau_{1}=\frac{1}{2 \pi \sqrt{-1}} \frac{d z}{z-z_{1}}, \quad \tau_{2}=\frac{1}{2 \pi \sqrt{-1}} \frac{d z}{z-z_{2}} .
$$

Suppose that, for $i=1,2, \gamma_{i}$ is a loop based at $z_{0}$ in $\mathbb{C}$ anti-clockwisely around $z_{i}$ which is nullhomotopic in $\mathbb{C}-\left\{z_{j}\right\}, j \neq i$. Then

$$
\begin{gathered}
\int_{\gamma_{1}} \frac{1}{2}\left[\tau_{1}, \tau_{2}\right]=\frac{1}{2 \pi \sqrt{-1}} \log (\lambda) \\
\int_{\gamma_{2}} \frac{1}{2}\left[\tau_{1}, \tau_{2}\right]=\frac{1}{2 \pi \sqrt{-1}} \log \left((1-\lambda)^{-1}\right) .
\end{gathered}
$$

Here $\lambda$ is the cross ratio

$$
\lambda=\left[z_{0}, z_{1}, z_{2}, \infty\right]=\frac{z_{0}-z_{2}}{z_{1}-z_{2}}
$$

and also

$$
(1-\lambda)^{-1}=\left[z_{2}, z_{0}, z_{1}, \infty\right]=\frac{z_{2}-z_{1}}{z_{0}-z_{1}}
$$

Proof. Since

$$
\int_{\gamma_{1}} \frac{d z}{z-z_{1}} \frac{d z}{z-z_{2}}=2 \pi \sqrt{-1} \log \left(\frac{z_{0}-z_{2}}{z_{1}-z_{2}}\right)
$$

and

$$
\int_{\gamma_{2}} \frac{d z}{z-z_{1}} \frac{d z}{z-z_{2}}=2 \pi \sqrt{-1} \log \left(\frac{z_{1}-z_{2}}{z_{0}-z_{2}}\right)
$$


then

$$
\begin{aligned}
\int_{\gamma_{1}}\left[\tau_{1}, \tau_{2}\right] & =\frac{1}{2 \pi \sqrt{-1}}\left(\log \left(\frac{z_{0}-z_{2}}{z_{1}-z_{2}}\right)-\log \left(\frac{z_{1}-z_{2}}{z_{0}-z_{2}}\right)\right) \\
& =\frac{1}{2 \pi \sqrt{-1}}\left(\log \left(\frac{z_{0}-z_{2}}{z_{1}-z_{2}}\right)^{2}\right) \\
& =2 \cdot \frac{1}{2 \pi \sqrt{-1}} \log \left(\frac{z_{0}-z_{2}}{z_{1}-z_{2}}\right) .
\end{aligned}
$$

From $\left[\tau_{1}, \tau_{2}\right]=-\left[\tau_{2}, \tau_{1}\right]$, we get

$$
\begin{aligned}
\int_{\gamma_{2}}\left[\tau_{1}, \tau_{2}\right] & =-\int_{\gamma_{2}}\left[\tau_{2}, \tau_{1}\right] \\
& =-2 \cdot \frac{1}{2 \pi \sqrt{-1}} \log \left(\frac{z_{0}-z_{1}}{z_{2}-z_{1}}\right) \\
& =2 \cdot \frac{1}{2 \pi \sqrt{-1}} \log \left(\frac{z_{2}-z_{1}}{z_{0}-z_{1}}\right) .
\end{aligned}
$$

COROLlary 3.2. Let $a_{1}, a_{2} \neq 0$ and

$$
\tau_{1}=\frac{1}{2 \pi \sqrt{-1}} \frac{a_{1} d z}{a_{1} z+b_{1}}, \quad \tau_{2}=\frac{1}{2 \pi \sqrt{-1}} \frac{a_{1} d z}{a_{2} z+b_{2}} .
$$

And $\gamma_{1}$ is a loop based at $z_{0}$ anti-clockwisely around $\left(-\frac{b_{1}}{a_{1}}\right)$ which is nullhomotopic in $\mathbb{C}-\left\{-\frac{b_{2}}{a_{2}}\right\}$. Then

$$
\int_{\gamma_{1}} \frac{1}{2}\left[\tau_{1}, \tau_{2}\right]=\frac{1}{2 \pi \sqrt{-1}} \log \left(\frac{a_{1}\left(a_{2} z_{0}+b_{2}\right)}{a_{1} b_{2}-b_{1} a_{2}}\right) .
$$

Proof. Set $z_{1}=-b_{1} / a_{1}, z_{2}=-b_{2} / a_{2}$. Applying to Lemma 5.1, we obtain

$$
\exp \left(2 \pi \sqrt{-1} \int_{\gamma_{1}} \frac{1}{2}\left[\tau_{1}, \tau_{2}\right]\right)=\frac{z_{0}-z_{2}}{z_{1}-z_{2}}=\frac{z_{0}+b_{2} / a_{2}}{-b_{1} / a_{1}+b_{2} / a_{2}}=\frac{a_{1}\left(a_{2} z_{0}+b_{2}\right)}{a_{1} b_{2}-b_{1} a_{2}} .
$$


Using this lemma, we can get the following proposition.

\section{Proposition 3.3.}

(1) For each $1 \leqq i<j \leqq n$ and $\gamma \in B H_{1}$,

$$
\int_{\gamma} \frac{1}{2}[\omega]_{i j}= \begin{cases}l\left(\lambda_{i j}\right) & \text { if } \gamma=\alpha_{i} \\ l\left(\left(1-\lambda_{i j}\right)^{-1}\right) & \text { if } \gamma=\alpha_{j} \\ l\left(\lambda_{i j}^{-1}\left(\lambda_{i j}-1\right)\right) & \text { if } \emptyset \neq H_{i} \cap H_{j} \subset G_{k} \text { and } \gamma=\beta_{k} \\ 0 & \text { otherwise. }\end{cases}
$$

(2) For each $1 \leqq i<j \leqq m$ and $\gamma \in B H_{1}$,

$$
\int_{\gamma} \frac{1}{2}[\eta]_{i j}= \begin{cases}l\left(\lambda_{i j}^{\prime}\right) & \text { if } \gamma=\beta_{i} \\ l\left(\left(1-\lambda_{i j}^{\prime}\right)^{-1}\right) & \text { if } \gamma=\beta_{j} \\ 0 & \text { otherwise }\end{cases}
$$

(3) For each $1 \leqq i \leqq j \leqq n, 1 \leqq k \leqq l \leqq m$ and $\gamma \in B H_{1}$,

$$
\begin{aligned}
& \int_{\gamma} \frac{1}{2}\{\omega\}_{i j}=\left\{\begin{array}{cc}
\frac{1}{2} & \text { if } i=j \text { and } \gamma=\alpha_{i} \\
0 & \text { otherwise, }
\end{array}\right. \\
& \int_{\gamma} \frac{1}{2}\{\eta\}_{k l}=\left\{\begin{array}{cc}
\frac{1}{2} & \text { if } k=l \text { and } \gamma=\beta_{k} \\
0 & \text { otherwise. }
\end{array}\right.
\end{aligned}
$$

For each $1 \leqq i \leqq n, 1 \leqq j \leqq m$ and $\gamma \in B H_{1}$,

$$
\int_{\gamma} \frac{1}{2}\left\{\omega_{i}, \eta_{j}\right\}=0
$$

Here

$$
\begin{gathered}
l(z)=\frac{1}{2 \pi \sqrt{-1}} \log (z), \\
\lambda_{i j}=\left[y_{0}, y_{i}, y_{j}, \infty\right]=\frac{y_{0}-y_{j}}{y_{i}-y_{j}},
\end{gathered}
$$

and

$$
\lambda_{i j}^{\prime}=\left[x_{0}, x_{i}, x_{j}, \infty\right]=\frac{x_{0}-x_{j}}{x_{i}-x_{j}} .
$$


Proof. (1) $\frac{1}{2}[\omega]_{\imath j}$ with $H_{i} \cap H_{\jmath}=\emptyset$. For each $1 \leqq s \leqq n$,

$$
\int_{\alpha_{s}} \omega_{i} \omega_{j}=\int_{\alpha_{s}} \frac{d y-d h_{i}(x)}{y-h_{i}(x)} \frac{d y-d h_{j}(x)}{y-h_{j}(x)}=\int_{\alpha_{s}} \frac{d y}{y-y_{\imath}} \frac{d y}{y-y_{j}} .
$$

Applying to Lemma 3.1, we get

$$
\int_{\alpha_{\imath}} \frac{1}{2}[\omega]_{i \jmath}=l\left(\left[y_{0}, y_{i}, y_{j}, \infty\right]\right)=l\left(\lambda_{\imath j}\right)
$$

and

$$
\int_{\alpha_{\jmath}} \frac{1}{2}[\omega]_{i j}=l\left(\left[y_{j}, y_{0}, y_{i}, \infty\right]\right)=l\left(\left(1-\lambda_{i \jmath}\right)^{-1}\right) .
$$

(1) $\frac{1}{2}[\omega]_{i j}$ with $\emptyset \neq H_{i} \cap H_{j} \subset G_{k}$. For each $1 \leqq s, t \leqq n$ and each $1 \leqq u \leqq m$, since

$$
\int_{\alpha_{s}}\left[\omega_{t}, \eta_{u}\right]=\int_{\alpha_{s}} \omega_{t} \eta_{u}-\int_{\alpha_{s}} \eta_{u} \omega_{t}=\int_{\alpha_{s}} \omega_{t}-\int_{\alpha_{s}} \omega_{t}=0
$$

we get

$$
\int_{\alpha_{s}}[\omega]_{i j}=\int_{\alpha_{s}}\left[\omega_{i}, \omega_{j}\right]
$$

Applying to Lemma 3.1, we obtain

$$
\int_{\alpha_{\imath}} \frac{1}{2}[\omega]_{i \jmath}=l\left(\left[y_{0}, y_{i}, y_{j}, \infty\right]\right)=l\left(\lambda_{i j}\right)
$$

and

$$
\int_{\alpha_{\jmath}} \frac{1}{2}[\omega]_{i j}=l\left(\left[y_{j}, y_{0}, y_{i}, \infty\right]\right)=l\left(\left(1-\lambda_{i j}\right)^{-1}\right) .
$$

For each $1 \leqq l \leqq m$,

$$
\int_{\beta_{l}}[\omega]_{i j}=\int_{\beta_{l}}\left[\omega_{i}, \omega_{\jmath}\right]+\int_{\beta_{l}}\left(\left[\omega_{j}, \eta_{k}\right]+\left[\eta_{k}, \omega_{i}\right]\right)=\int_{\beta_{l}}\left[\omega_{j}, \eta_{k}\right]+\int_{\beta_{l}}\left[\eta_{k}, \omega_{i}\right]
$$

and for $l \neq k$

$$
\int_{\beta_{l}}[\omega]_{\imath j}=0
$$

Then it is enough to compute

$$
\int_{\beta_{k}}\left[\eta_{k}, \omega_{i}\right]
$$


Set

$$
\begin{array}{ll}
H_{i}: & y=a_{i} x+b_{i} \\
H_{j}: & y=a_{j} x+b_{j} \\
G_{k}: & \left(a_{i}-a_{j}\right) x+\left(b_{i}-b_{j}\right)=0 .
\end{array}
$$

By means of Corollary 3.2 we get

$$
\begin{aligned}
\int_{\beta_{k}} \frac{1}{2}\left[\eta_{k}, \omega_{i}\right] & =\int_{\beta_{k}} \frac{1}{2}\left[\frac{1}{2 \pi \sqrt{-1}} \frac{\left(a_{i}-a_{j}\right) d x}{\left(a_{i}-a_{j}\right) x+\left(b_{i}-b_{j}\right)}, \frac{1}{2 \pi \sqrt{-1}} \frac{a_{i} d x}{a_{i} x+b_{i}-y_{0}}\right] \\
& =\frac{1}{2 \pi \sqrt{-1}} \log \left(\frac{\left(a_{2}-a_{j}\right)\left(a_{i} x_{0}+b_{i}-y_{0}\right)}{\left(a_{i}-a_{j}\right)\left(b_{i}-y_{0}\right)-a_{i}\left(b_{i}-b_{\jmath}\right)}\right) \\
& =\frac{1}{2 \pi \sqrt{-1}} \log \left(\frac{\left(a_{i}-a_{j}\right)\left(y_{i}-y_{0}\right)}{a_{i} b_{j}-\left(a_{i}-a_{j}\right) y_{0}}\right)
\end{aligned}
$$

and, in the same way,

$$
\int_{\beta_{k}} \frac{1}{2}\left[\eta_{k}, \omega_{j}\right]=\frac{1}{2 \pi \sqrt{-1}} \log \left(\frac{\left(a_{i}-a_{j}\right)\left(y_{j}-y_{0}\right)}{a_{i} b_{j}-\left(a_{i}-a_{j}\right) y_{0}}\right) .
$$

Consequently we obtain

$$
\begin{aligned}
\int_{\beta_{k}} \frac{1}{2} \omega_{i j} & =l\left(\frac{\left(a_{i}-a_{j}\right)\left(y_{i}-y_{0}\right)}{a_{i} b_{j}-\left(a_{i}-a_{j}\right) y_{0}} \cdot \frac{a_{i} b_{j}-\left(a_{i}-a_{j}\right) y_{0}}{\left(a_{i}-a_{j}\right)\left(y_{i}-y_{0}\right)}\right) \\
& =l\left(\frac{y_{i}-y_{0}}{y_{j}-y_{0}}\right)=l\left(\left[y_{i}, y_{0}, y_{j}, \infty\right]\right)=l\left(\lambda_{i j}^{-1}\left(\lambda_{i j}-1\right)\right) .
\end{aligned}
$$

(2) $\frac{1}{2}[\eta]_{i j}$. We apply $\eta_{i}, \eta_{j}, \beta_{i}, \beta_{j}$ to Lemma 3.1 and then get

$$
\int_{\beta_{\imath}} \frac{1}{2} \eta_{i j}=l\left(\left[x_{0}, x_{i}, x_{j}, \infty\right]\right)=l\left(\lambda_{i j}^{\prime}\right)
$$

and

$$
\int_{\beta_{j}} \frac{1}{2} \eta_{i j}=l\left(\left[x_{j}, x_{0}, x_{i}, \infty\right]\right)=l\left(\left(1-\lambda_{i j}^{\prime}\right)^{-1}\right) .
$$

(3) $\frac{1}{2}\{$,$\} . In general, the shuffle formula of iterated integrals (see$ $[\mathrm{H} 1 ;(2.11)])$ gives

$$
\int_{\gamma}\{\omega, \eta\}=\int_{\gamma} \omega \eta+\int_{\gamma} \eta \omega=\int_{\gamma} \omega \int_{\gamma} \eta .
$$


By the duality between $\omega_{i}, \eta_{k}$, and $\left[\alpha_{\imath}\right],\left[\beta_{k}\right]$, for any braces basis $\frac{1}{2}\{\omega, \eta\}$ of $K$ with $\omega \neq \eta$ and $[\gamma] \in\left\{\left[\alpha_{\imath}\right],\left[\beta_{k}\right]\right\}$, we get

$$
\int_{\gamma} \frac{1}{2}\{\omega, \eta\}=0 .
$$

For any braces base $\frac{1}{2}\{\omega, \omega\}$ of $K$ and a dual base $[\gamma]$ of $\omega$, we get

$$
\int_{\gamma} \frac{1}{2}\{\omega, \omega\}=\frac{1}{2}\left(\int_{\gamma} \omega\right)^{2}=\frac{1}{2} .
$$

And also for $\left[\gamma^{\prime}\right] \neq[\gamma]$,

$$
\int_{\gamma^{\prime}} \frac{1}{2}\{\omega, \omega\}=0
$$

COROLlary 3.4. The extension isomorphism $\psi$ associated with the mixed Hodge structure on $\left(J / J^{3}\right)^{*}$ is

$$
\begin{aligned}
& \psi\left(\left(J / J^{3}\right)^{*}\right)= \sum_{(i, j, k) \in C} l\left(\lambda_{\imath \jmath}\right)\left[\alpha_{i}-1, \alpha_{j}-1\right] \otimes\left(\alpha_{\imath}-1\right)^{*} \\
&+l\left(\left(1-\lambda_{i j}\right)^{-1}\right)\left[\alpha_{i}-1, \alpha_{\jmath}-1\right] \otimes\left(\alpha_{j}-1\right)^{*} \\
&+l\left(\lambda_{i j}^{-1}\left(\lambda_{i j}-1\right)\right)\left[\alpha_{i}-1, \alpha_{j}-1\right] \otimes\left(\beta_{k}-1\right)^{*} \\
&+ \sum_{(i, j) \in P} l\left(\lambda_{i j}\right)\left[\alpha_{i}-1, \alpha_{j}-1\right] \otimes\left(\alpha_{i}-1\right)^{*} \\
&+ \quad \sum_{1 \leqq l<j \leqq m} l\left(\left(1-\lambda_{i j}^{\prime}\right)\left[\beta_{i}-1, \beta_{j}-1\right] \otimes\left(\beta_{\imath}-1\right)^{*}\right. \\
& \quad+l\left(\left(1-\alpha_{i j}-1, \alpha_{j}-1\right] \otimes\left(\alpha_{j}-1\right)^{*}\right)\left[\beta_{i}-1, \beta_{j}-1\right] \otimes\left(\beta_{j}-1\right)^{*} \\
& \bmod \left(K^{*} \otimes H_{1}^{*}\right)_{\mathbb{Z}}
\end{aligned}
$$

where

$$
C=C(\mathcal{A})=\left\{(i, j, k) \mid 1 \leqq i<j \leqq n, \emptyset \neq H_{\imath} \cap H_{j} \subset G_{k}\right\}
$$

and

$$
P=P(\mathcal{A})=\left\{(i, j) \mid 1 \leqq i<j \leqq n, H_{i} \cap H_{\jmath}=\emptyset\right\}
$$


Proof. In order to prove this we compute its motif. According to $\S 2$, there is an extension

$$
0 \longrightarrow H^{1}(M) \longrightarrow K \longrightarrow\left(J(M, b) / J^{3}\right)^{*} \longrightarrow 0
$$

of $\left(J(M, b) / J^{3}\right)^{*}$. Since $H^{1}(M)$ is a pure Hodge structure of weight 2 its motif is

$$
\mu: K_{\mathbb{Z}} \rightarrow H_{\mathbb{C}}^{1}(M) / H_{\mathbb{Z}}^{1}(M) .
$$

The exponential map gives a canonical identification

$$
\begin{gathered}
H_{\mathbb{C}}^{1}(M) / H_{\mathbb{Z}}^{1}(M) \rightarrow\left(\mathbb{C}^{*}\right)^{n} \times\left(\mathbb{C}^{*}\right)^{m} \\
\sum a_{i} \omega_{i}+\sum b_{j} \eta_{j} \rightarrow\left(\exp 2 \pi \sqrt{-1} a_{i}, \exp 2 \pi \sqrt{-1} b_{\jmath}\right) .
\end{gathered}
$$

Thus, for $z_{1} \otimes z_{2} \in K$ and $[\gamma] \in H_{1}(M)$,

$$
\begin{gathered}
\mu\left(z_{1} \otimes z_{2}\right)[\gamma]=\exp 2 \pi \sqrt{-1} \int_{\gamma} z_{1} z_{2} . \\
\omega_{i j}= \begin{cases}\frac{1}{2}[\omega]_{i j}+\frac{1}{2}\{\omega\}_{i j}+\frac{1}{2}\left\{\omega_{\imath}, \eta_{k}\right\}+\frac{1}{2}\left\{\omega_{\jmath}, \eta_{k}\right\} & \text { if } \emptyset \neq H_{i} \cap H_{j} \subset G_{k} \\
\frac{1}{2}[\omega]_{i j}+\frac{1}{2}\{\omega\}_{i j} & \text { if } H_{i} \cap H_{j}=\emptyset\end{cases}
\end{gathered}
$$

and

$$
\eta_{i j}=\frac{1}{2}[\eta]_{i j}+\frac{1}{2}\{\eta\}_{i j}
$$

Then, using the proposition 3.3, we can compute values of a basis $B K_{\mathbb{Z}}$ of $K_{\mathbb{Z}}$ obtained in $\S 2$.

Remark 3.5. Using

$$
\int_{\gamma} \omega \eta+\int_{\gamma} \eta \omega=\int_{\gamma} \omega \int_{\gamma} \eta
$$

we get

$$
\int_{\gamma} \frac{1}{2}[\omega, \eta]=\int_{\gamma} \omega \eta-\frac{1}{2} \int_{\gamma} \omega \int_{\gamma} e t a .
$$

It leads

$$
\int_{\alpha_{\imath}} \omega_{i} \omega_{j}=\int_{\alpha_{i}} \frac{1}{2}\left[\omega_{i}, \omega_{j}\right]=-\int_{\alpha_{\imath}} \omega_{j} \omega_{i}
$$

From this fact and the above proposition we also obtain the corollary.

This corollary leads the following definition and theorem. 
Definition 3.6. Let $\mathcal{A}$ be a fiber type 2-arrangement and $b$ a base point of $M(\mathcal{A})$. A pair $(\mathcal{A}, b)$ is called a pointed fiber type 2-arrangement. Two pointed fiber type 2-arrangements $(\mathcal{A}, b)$ and $\left(\mathcal{A}^{\prime}, b^{\prime}\right)$ are cross ratio equivalent if there is a one-to-one correspondence between $\mathcal{A}$ and $\mathcal{A}^{\prime}$ satisfying following conditions (1), (2) and (3); suppose that

$$
\mathcal{A}=\left\{H_{1}, \cdots, H_{n}, G_{1}, \cdots, G_{m}\right\}
$$

and

$$
\mathcal{A}^{\prime}=\left\{H_{1}^{\prime}, \cdots, H_{n}^{\prime}, G_{1}^{\prime}, \cdots, G_{m}^{\prime}\right\}
$$

where $H_{i}^{\prime}, G_{j}^{\prime}$ are corresponded to $H_{i}, G_{j}$, respectively.

(1) $H_{i} \cap H_{j}=\emptyset \Longleftrightarrow H_{i}^{\prime} \cap H_{j}^{\prime}=\emptyset$

(2) $\emptyset \neq H_{i} \cap H_{j} \subset G_{k} \Longleftrightarrow \emptyset \neq H_{i}^{\prime} \cap H_{j}^{\prime} \subset G_{k}^{\prime}$

$$
\lambda_{i j}(\mathcal{A}, b)=\lambda_{i j}\left(\mathcal{A}^{\prime}, b^{\prime}\right)
$$

and

$$
\lambda_{i j}^{\prime}(\mathcal{A}, b)=\lambda_{\imath j}^{\prime}\left(\mathcal{A}^{\prime}, b^{\prime}\right)
$$

Consequently we obtain the following theorem.

THEOREM 3.7. Let $(\mathcal{A}, b)$ and $\left(\mathcal{A}^{\prime}, b^{\prime}\right)$ be pointed fiber type 2-arrangements. If there is a ring isomorphism

$$
\varphi: \mathbb{Z} \pi_{1}(M(\mathcal{A}), b) / J^{3} \rightarrow \mathbb{Z} \pi_{1}\left(M\left(\mathcal{A}^{\prime}\right), b^{\prime}\right) / J^{3}
$$

which induces an isomorphism of mixed Hodge structures, then $(\mathcal{A}, b)$ and $\left(\mathcal{A}^{\prime}, b^{\prime}\right)$ are cross ratio equivalent.

Proof. The isomorphism $\varphi$ of mixed Hodge structures induces an isomorphism of Hodge structures on $W_{-1} / W_{-2}=J / J^{2} \cong H_{1}$, on $H^{1}$ and on $K=\operatorname{Ker}\left(H^{1} \otimes H^{1} \rightarrow H^{2}\right)$. Thus there is a one-to-one correspondence between $\mathcal{A}$ and $\mathcal{A}^{\prime}$ satisfying conditions (1), (2). And also it induces a congruent class of extensions

$$
0 \longrightarrow H^{1}(M) \longrightarrow K \longrightarrow 0
$$

of $K$ by $H^{1}$. Hence, according to Corollary 3.4, we get $\lambda_{i j}(\mathcal{A}, b)=\lambda_{i j}\left(\mathcal{A}^{\prime}, b^{\prime}\right)$ and $\lambda_{\imath \jmath}^{\prime}(\mathcal{A}, b)=\lambda_{i j}^{\prime}\left(\mathcal{A}^{\prime}, b^{\prime}\right)$.

Acknowledgements. The author would like to thank Professor T. Terasoma for his helpful suggestions and for valuable discussions. 


\section{REFERENCES}

[B] E. Brieskorn, Sur les groupes de tresses, Séminarire Bourbaki Lecture Notes in Math., 317, Springer (1973), p. 21-44.

[Ca] J. A. Carlson, Extensions of mixed Hodge structures, Journées de Géometrie Algébrique d'Angers (A.Beauville, ed.), Sijthoff and Noordhoff, Alphen aan den Rijn (1980), p. 77-105.

[C1] K.-T. Chen, Algebras of tterated path integrals and fundamental groups, Trans. Amer. Math. Soc., 156 (1971), 359-379.

[C2] — Extension of $C^{\infty}$ function algebra by integrals and Malcev completion of $\pi_{1}$, Adv. in Math, 23 (1977), 181-210.

[C3] - Iterated path integrals, Bull.Amer. Math.Soc., 83 (1977), 831-879.

[D] P. Deliegne, Théorie de Hodge II, Publ.Math. I.H.E.S., 40 (1971), 5-58.

[Du] A. Durfee, A naive guide to mixed Hodge theory, Singularities, Proc.Sympo. Pure Math. (R.I. Providence, ed.), 40 (part 1), Amer. Math. Soc. (1983), p. 313-320.

[E] F. El Zein, Introduction à la théorie de Hodge mixte, HERMANN ÉDITEUR DES SCIENCES ET DES ARTS, 1991.

[FR] M. Falk and R. Randell, The lower central series of a fiber-type arrangement, Invent. Math., 82 (1985), 77-88.

[GS] P. Griffiths and W. Schmid, Recent developments in Hodge theory: A discussion of techniques and results, Proc. Bombay Colloq. on Discrete Subgroups of Lie Groups (Bombay,1973), Oxfold Univ. Press (1975), p. 31-127.

[H1] R. M. Hain, The geometry of the mixed Hodge structure on the fundamental group, Algebraic geometry Amer. Math. Soc. Bowdoin, Proc. Symp. Pure Math., 46 (1987), p. 247-282.

[H2] - On the generalization of Hilbert's 21st problem, Ann. Sci. Ec. Norm.Super., IV (1986), 609-627.

[H3] - Iterated integrals and mixed Hodge structures on homotopy groups, Hodge theory Lecture Notes in Math., 1246, Springer (1987), p. 75-83.

[H4] - Higher Albanese manifolds, Hodge theory Lecture Notes in Math., 1246, Springer (1987), p. 84-91.

[H5] - The de Rham homotopy theory of complex algebraic varieties I, $K$-Theory, 1 (1987), 271-324.

[HZ1] R. M. Hain and S. Zucker, Unipotent variations of mixed Hodge structure, Invent.math., 88 (1987), 83-124.

[HZ2] _ A guide to unipotent variatıons of mixed Hodge structure, Hodge theory Lecture Notes in Math., 1246, Springer (1987), p. 92-106.

[J] M . Jambu, Fiber-type arrangements and factorization properties, Adv. in Math., 80 (1990), 1-21.

[K1] T. Kohno, On the holonomy Lie algebra and the nilpotent completion of the fundamental group of the complement of hypersurfaces, Nagoya Math. J., 92 (1983), 21-37.

[K2] Holonomy Lie algebras, logarthmic connections and the lower central series of fundamental groups, Cont. Math., 90 (1989), 171-182. 
[K3] — Integrable connections related to Manin and Schechtman's higher braid groups, Illinois J.Math., 34 (1990), 476-484.

[M] J. W. Morgan, The algebraic topology of smooth algebranc varieties, Publ.Math. I.H.E.S., 48 (1978), 137-204.

[OT] P. Orlik and H. Terao, Arrangements of Hyperplanes, Grundlehren der mathematischen Wissenschaften 300, Springer-Verlag, 1992.

[Sh] B. Z. Shapiro, The mixed Hodge structure of the complement to an arbitrary arrangement of affine complex hyperplanes is pure, Proc. Amer. Math. Soc., 117 (1993), 931-933.

Department of Mathematical Sciences

University of Tokyo

Komaba, Meguro-ku, Tokyo 153

Japan

CURRENT ADDRESS:

Department of Mathematics

Tokyo Metropolitan Unvversity

Minami-ohsawa, Hachioji 192-03

Japan

kawahara@math.metro-u.ac.jp 